\title{
AVR1-CO39 Is a Predominant Locus Governing the Broad Avirulence of Magnaporthe oryzae 2539 on Cultivated Rice (Oryza sativa L.)
}

\author{
Yan Zheng, ${ }^{1}$ Wenhui Zheng, ${ }^{2}$ Fucheng Lin, ${ }^{3}$ Ying Zhang, ${ }^{2}$ Yunping Yi, ${ }^{2}$ Baohua Wang, ${ }^{2}$ Guodong Lu, ${ }^{2}$ \\ Zonghua Wang, ${ }^{2,4}$ and Weiren $\mathrm{Wu}^{1,4}$ \\ ${ }^{1}$ Key Laboratory of Ministry of Education for Genetics, Breeding and Multiple Utilization of Crops, and ${ }^{2}$ Key Laboratory of \\ Ministry of Education for Biopesticide and Chemical Biology, Fujian Agriculture \& Forestry University, Fuzhou, Fujian, \\ 350002, China; ${ }^{3}$ Institute of Biotechnology, College of Agriculture \& Biotechnology, Zhejiang University, Hangzhou, \\ Zhejiang, 310029, China; ${ }^{4}$ School of Life Sciences, Fujian Agriculture \& Forestry University, Fuzhou, Fujian, 350002, China \\ Submitted 23 October 2009. Accepted 8 September 2010.
}

\begin{abstract}
Magnaporthe oryzae 2539 was previously found to be avirulent to most rice cultivars and, therefore, was assumed to carry many avirulence $(A V R)$ genes. However, only one $A V R$ gene, $A V R 1-C O 39$, which corresponds to a resistance (R) gene $\mathrm{Pi}$-CO39(t) in rice cv. CO39, has been found from 2539 thus far. In order to identify more $A V R$ genes, we isolated 228 progeny strains from a cross between 2539 and Guy11, an $M$. oryzae strain with strong virulence on rice, and inoculated these strains onto 23 rice accessions (22 individual cultivars and a mixture of 14 cultivars) that are all resistant to 2539 but susceptible to Guy11. Unexpectedly, the experimental results indicated that the avirulence of 2539 on these rice cultivars appeared to be controlled only by the $A V R 1-C O 39$ locus. Consistent with this result, we further found that all except one of the rice cultivars were resistant to two transformed Guy11 strains carrying a 1.05-kb fragment containing the $A V R 1-C O 39$ gene from 2539. These results suggest that $A V R 1-C O 39$ is a predominant locus controlling the broad avirulence of 2539 on cultivated rice. Based on the results of this study and other previous studies, we infer that $A V R 1-C O 39$ is a species-wise rather than a cultivar-wise host-specific $A V R$ locus of $M$. oryzae for rice.
\end{abstract}

\footnotetext{
Magnaporthe oryzae is the causal fungus of rice blast, one of the most economically devastating diseases of rice worldwide, and can also infect many other gramineous plants (Couch and Kohn 2002). It has been known that fungal avirulence on rice is controlled by some major genes (called avirulence $[A V R]$ genes) that correspond to specific rice resistance $(R)$ genes following a gene-for-gene relationship (Silué et al. 1992). AVR genes determine specific gene expressions and functions in host cultivars. Loss or mutation of $A V R$ genes may lead to the generation of new pathogenicity and the loss of corresponding host resistance. Therefore, research of $A V R$ genes
}

Corresponding authors: W. Wu; Telephone and Fax: +86-591-83789176, E-mail: wuwr@fjau.edu.cn; and Z. Wang; Telephone and Fax: +86-59183789202; E-mail: wangzh@ fjau.edu.cn

Y. Zheng and W. Zheng contributed equally to this work.

* The $\boldsymbol{e}$-Xtra logo stands for "electronic extra" and indicates that a supplementary figure is published online. is important for understanding the biological mechanisms of interactions between hosts and pathogens.

To date, many $M$. oryzae AVR genes have been identified and mapped. However, only eight $A V R$ genes - $P W L 2$ (Sweigard et al. 1995), AVR1-CO39 (Farman and Leong 1998), AVR-Pita (Orbach et al. 2000), ACEl (Bohnert et al. 2004), AvrPiZ- $t$ (Li et al. 2009), AVR-Pia (Miki et al. 2009), AVR-Pii, and AVR$P i k / k m / k p$ (Yoshida et al. 2009) — have been cloned and only one example of direct interaction between a rice $\mathrm{R}$ protein (Pi-ta) and an $M$. oryzae AVR protein (AVR-Pita) has been known (Jia et al. 2000).

AVR1-CO39, a gene specifying avirulence on rice cv. CO39, was first identified in M. oryzae 4091-5-8 (Valent et al. 1991) and subsequently in strain 2539 (Smith and Leong 1994), and was then cloned from 2539 by map-based cloning (Farman and Leong 1998). The avirulence function of AVR1-CO39 was assumed to depend on a resistance gene, $\mathrm{Pi}$-CO39(t), in CO39 following the gene-for-gene interaction mechanism (Chauhan et al. 2002). It was reported that an intact AVRI-CO39 locus was not present in any of the $M$. oryzae isolates from rice but relatively stable and conserved among most non-rice-infecting $M$. oryzae isolates; this explained why most $M$. oryzae isolates from rice were virulent on CO39 (Farman et al. 2002; Tosa et al. 2005).

Isolate 2539 is a laboratory strain of $M$. oryzae with a complex pedigree consisting of fertile isolates from goosegrass, weeping lovegrass, finger millet, two Japanese rice isolates, two Chinese rice isolates, and two laboratory isolates not pathogenic on rice (Leung et al. 1988). The AVRl-CO39 gene in 2539 was inherited from strain 4091-5-8, which inherited AVRI-CO39 from K76-79, an isolate from weeping lovegrass (Farman and Leong 1998; Leung et al. 1988; Valent et al. 1986). Smith and Leong (1994) systematically investigated the pathogenicity of 2539 on rice using 765 rice accessions. They found that most of the rice accessions were not infected by 2539 ; only $29(3.8 \%)$ accessions were moderately susceptible to 2539 . To explain this result, they assumed that 2539 might be a rich source of $A V R$ genes specific to different rice cultivars (Leong et al. 1994).

The above assumption implies that it would be possible to uncover a number of different $A V R$ genes in 2539. Therefore, we tried to identify some $A V R$ genes other than AVRI-CO39 in 2539. However, our results appeared to negate the hypothesis that many avirulence genes exist in 2539 but suggest that the AVR1-CO39 locus predominantly confers the general avirulence of 2539 on rice. 


\section{RESULTS}

Genetic association between the AVR1-CO39 locus and 2539 avirulence on different rice cultivars.

A population consisting of 228 progeny strains from a cross between the isolate 2539 and highly pathogenic strain Guy11 was constructed. Polymerase chain reaction (PCR) analysis indicated that 107 strains carried the avirulence gene AVRI-CO39 and 121 strains did not (Fig. 1). This was consistent with the theoretical $(1: 1)$ segregation ratio $\left(\chi^{2}=0.86, P=0.354\right)$. Three inoculation experiments were conducted using a total of 23 rice cultivars (Table 1), which are all resistant to 2539 but susceptible to Guy11 according to our preparatory experiment. In the first experiment, all 228 strains were used to inoculate five rice cultivars (Table 1 , numbers 1 to 5 ). $\mathrm{Cv}$. $\mathrm{CO} 39$ was proposed to carry the $R$ gene $P i-C O 39(t)$, corresponding to AVRI-CO39 (Chauhan et al. 2002). Hence, it could be expected that the strains carrying AVRl-CO39 and those lacking AVRl-CO39 would be avirulent and virulent, respectively, on CO39. This was validated for most of the strains in the experiment; only a small proportion of the strains exhibited inconsistent phenotypes (Table 2). The results suggested that the inoculation was reliable in general, although experimental error existed to some extent.

For cvs. 2 to 5 , the strains also showed 1 (virulence) to 1 (avirulence) segregation, suggesting that there should be an $A V R$ gene for each of the cultivars. To our surprise, the responses of these cultivars to the strains were highly consistent with those of CO39. Almost all the strains virulent on CO39 were also virulent on the four cultivars (although the symptom was less serious in Nipponbare, Minghui63, and Zhenshan97 but more serious in DZ60 in comparison with CO39) and all the strains avirulent on CO39 were also avirulent on the four cultivars. The results suggest that the avirulence of 2539 on these four cultivars also should be owing to the AVR1-CO39 locus. Because the five cultivars used in the first experiment were from different sources, the above results imply that AVR1-CO39 might be a locus with general avirulence on cultivated rice in 2539 .

To validate the above results and inference, we conducted a second inoculation experiment on cvs. 1 to 5 and an additional 10 cultivars (Table 1 , numbers 6 to 15 ) using 52 strains (11 carrying AVRl-CO39 and 41 lacking AVRl-CO39) randomly selected from the 228 progeny strains. For cvs. 6 to 15 , only parts of the 41 strains lacking AVRl-CO39 were investigated because some pots of seedlings of these cultivars died before inoculation. The phenotypes of the 52 strains on cvs. 1 to 5 were all consistent with their AVR1-CO39 genotypes as expected according to the results obtained in the first experiment; namely, all 11 strains carrying AVR1-CO39 could not but all 41 strains not carrying AVRI-CO39 could infect the five cultivars (Table 2 ). For the 10 additional cultivars, the observed pathogenicity of the tested strains was also consistent with their AVRI-CO39 genotypes except that a few (one to four) strains lacking $A V R 1$ -
CO39 appeared to be avirulent on cvs. 12 to 15 (Table 2); these exceptional strains were later proven to be able to infect the four cultivars by reexamination in a supplemental inoculation experiment. Hence, the avirulence of 2539 on the 10 additional cultivars tested should also impute the AVRI-CO39 locus. These results support the inference that the AVRl-CO39 locus confers the general avirulence of 2539 on cultivated rice.

However, in the above inoculation experiments, most of the rice cultivars used belonged to indica subspecies and were from China. Hence, more evidence may still be required for the above inference. For this purpose, we performed a third inoculation experiment with a number of japonica rice cultivars from nine foreign countries (Table 1, numbers 16 to 23). Accession number 23 was a mixture of an equal number of seed of 14 japonica rice cultivars, which did not have sufficient seed available each for the inoculation experiment individually. In all, 47 progeny strains (20 carrying and 27 lacking AVRl-CO39) were randomly selected for the experiment. As expected, the strains lacking AVRI-CO39 appeared to be virulent on all of these rice cultivars, whereas those carrying AVRl-CO39 appeared to be avirulent (Table 2). Thus, all of the results of the three inoculation experiments were consistent. Taken together, the above genetic analysis results strongly suggest that the single locus AVR1-CO39 controls a broad avirulence on cultivated rice.

Table 1. Rice cultivars used for the experiment

\begin{tabular}{llll}
\hline No. & Name or code & Type & Origin \\
\hline 1 & CO39 & Indica & India \\
2 & Nipponbare & Japonica & Japan \\
3 & DZ60 & Javanica & Bangladesh \\
4 & Minghui63 & Indica & China \\
5 & Zhenshan97B & Indica & China \\
6 & JXR80 & Indica & China \\
7 & Kehui752 & Indica & China \\
8 & Shen3B & Japonica & China \\
9 & K1713 & Indica & China \\
10 & ZhongjiuB & Indica & China \\
11 & Hua1B & Indica & China \\
12 & D62B & Indica & China \\
13 & Gang46B & Indica & China \\
14 & JinshanB3 & Indica & China \\
15 & T80B & Indica & China \\
16 & IRBLks-F5 & Japonica & Japan \\
17 & IRBLsh-S & Japonica & Japan \\
18 & IRBLta-CP1 & Japonica & Philippines \\
19 & IRBL12-M & Japonica & Philippines \\
20 & Lement & Japonica & United States \\
21 & Dular & Indica & India \\
22 & P183 & Indica & Indonesia \\
23 & Mixture & Japonica & Multiple \\
\hline
\end{tabular}

${ }^{a}$ Containing an equal number of seeds of 14 japonica rice cultivars from America (Blue Belle and Peeso), Bulgaria (MC, Myxak, and 04-329), Italy (3224 and 8068), Japan (Akita Komochi, Toyonishiki, and 05-394), Korea (WL060097), and Russia (Спроги, Узрос 269, and UZ Ros 72).

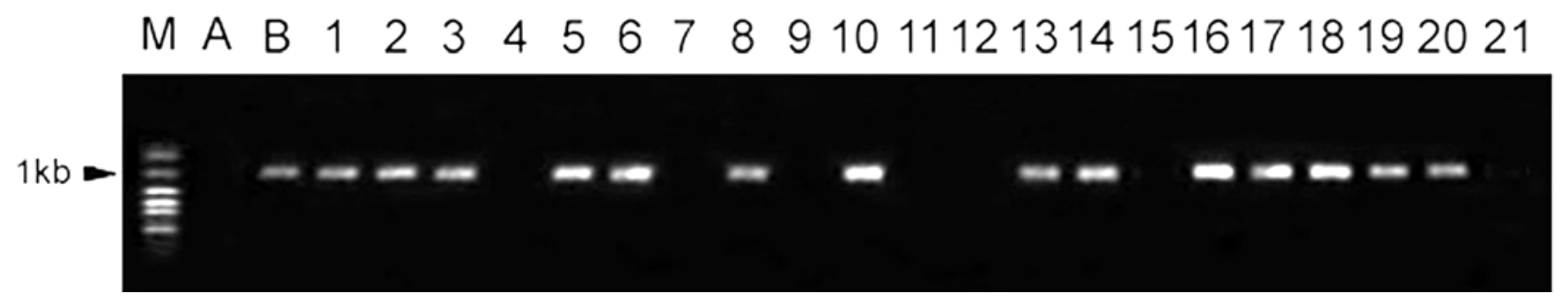

Fig. 1. Detection of the AVR1-CO39 gene in different Magnaporthe oryzae strains by polymerase chain reaction using primers AVF1-2 and AVR1-2. A 1.05-kb band was present when the target gene existed. $\mathrm{M}=$ molecular weight marker, $\mathrm{A}=$ Guy $11, \mathrm{~B}=2539,1 \mathrm{anes} 1$ to $3,5,6,8,10,13,14$, and 16 to $20=$ avirulent strains, and lanes $4,7,9,11,12,15$, and $21=$ virulent strains. 
Pathogenicity of AVR1-CO39-transformed Guy11 strains.

To examine whether the general avirulence of 2539 on rice associated with the AVR1-CO39 locus is controlled by the AVRl-CO39 gene or there are any other AVR genes at this locus, we isolated gene AVRI-CO39 from 2539 and transferred it into Guy11. Six transformants were acquired and two of them, PCX62-55 and PCX62-64 (Fig. 2), were used for an inoculation experiment. In order to validate the presence and integrity of AVR1-CO39 in these transformants, we amplified and sequenced the fragment $(1.70 \mathrm{~kb})$ containing AVRl-CO39 from transformants and compared them with the original sequence of AVR1-CO39 from $2539(1.05 \mathrm{~kb})$. The result indicated that these two sequences were the same in the $1.05-\mathrm{kb}$ overlapped region except for two synonymous mutations (Supplementary Fig. 1), confirming that the transformants were positive. Inoculation on detached leaves and seedlings indicated that the two transformants could not infect all the rice cultivars tested (Table 1), except for Shen3B, suggesting that AVR1-CO39 gene should be the cause of the avirulence of 2539 on all the rice cultivars tested except for Shen3B, for which there might be another $A V R$ gene tightly linked to the AVRI-CO39 gene (or located at the same locus).

\section{DISCUSSION}

Smith and Leong (1994) inoculated a large number (765) of rice lines with $M$. oryzae 2539 . They found that $96.2 \%$ (736/765) of the rice lines could not be infected by 2539 . According to this result, it was presumed that 2539 might carry many cultivar specificity genes (Leong et al. 1994). If this assumption were true, 2539 would be a very valuable material for cloning $A V R$ genes in $M$. oryzae as well as corresponding $R$ genes in rice and for studying the molecular mechanisms of interactions between $A V R$ and $R$ genes. However, only one $A V R$ gene (AVRl-CO39) has been identified in 2539 thus far. In this study, we intended to identify more putative $A V R$ genes

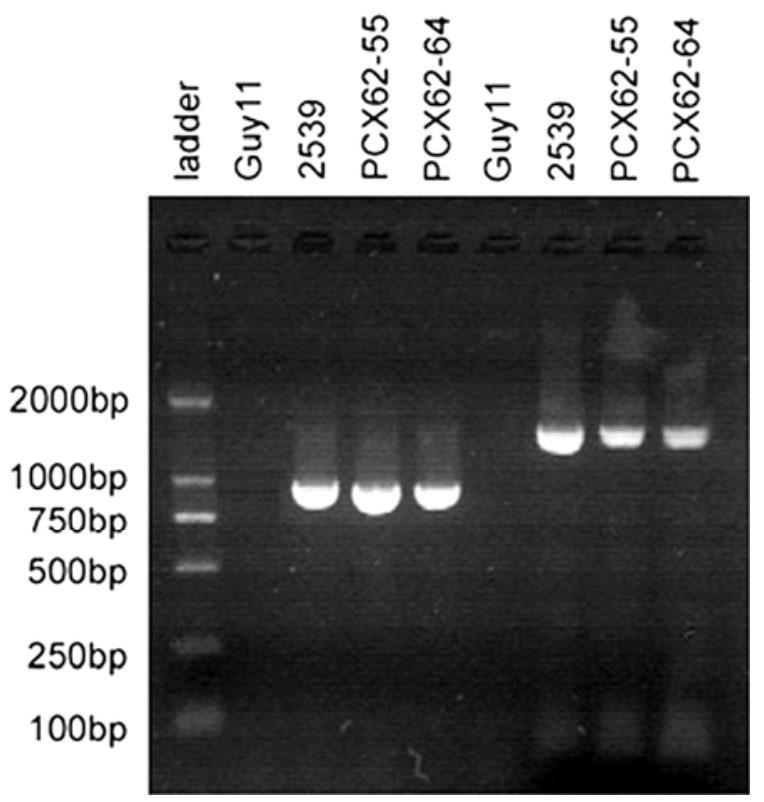

Fig. 2. Detection of the AVR1-CO39 gene in transformants PCX62-55 and PCX62-64 by polymerase chain reaction. Lane 1, molecular weight marker D2000 (TIANGEN); lanes 2 to 5, fragment (1.05 kb) amplified by primers AVF1-2 and AVR1-2; lanes 6 to 9, fragment (1.70 kb) amplified by primers WF6 and WR4.

Table 2. Identification of AVR1-CO39 genotypes of Magnaporthe oryzae strains and their pathogenicities on different rice cultivars

\begin{tabular}{|c|c|c|c|c|c|}
\hline \multirow[b]{2}{*}{ No. } & \multirow[b]{2}{*}{ Cultivar } & \multicolumn{2}{|c|}{ AVR1-CO39 (+) } & \multicolumn{2}{|c|}{ AVR1-CO39 (-) } \\
\hline & & Virulent & Avirulent & Virulent & Avirulent \\
\hline \multicolumn{6}{|c|}{ Experiment I } \\
\hline 1 & CO39 & 5 & 104 & 116 & 3 \\
\hline 2 & Nipponbare & 3 & 103 & 111 & 5 \\
\hline 3 & DZ60 & 2 & 103 & 114 & 3 \\
\hline 4 & Minhui63 & 4 & 104 & 112 & 4 \\
\hline 5 & Zhenshan97B & 5 & 102 & 111 & 5 \\
\hline \multicolumn{6}{|c|}{ Experiment II } \\
\hline 1 & $\mathrm{CO} 39$ & 0 & 11 & 41 & 0 \\
\hline 2 & Nipponbare & 0 & 11 & 41 & 0 \\
\hline 3 & DZ60 & 0 & 11 & 41 & 0 \\
\hline 4 & Minhui63 & 0 & 11 & 41 & 0 \\
\hline 5 & Zhenshan97B & 0 & 11 & 41 & 0 \\
\hline 6 & JXR80 & 0 & 11 & 28 & 0 \\
\hline 7 & Kehui752 & 0 & 11 & 29 & 0 \\
\hline 8 & Shen3B & 0 & 11 & 40 & 0 \\
\hline 9 & K1713 & 0 & 11 & 32 & 0 \\
\hline 10 & ZhongjiuB & 0 & 11 & 35 & 0 \\
\hline 11 & Hua1B & 0 & 11 & 32 & 0 \\
\hline 12 & D62B & 0 & 11 & 26 & 1 \\
\hline 13 & Gang46B & 0 & 11 & 30 & 2 \\
\hline 14 & JinshanB3 & 0 & 11 & 26 & 3 \\
\hline 15 & $\mathrm{~T} 80 \mathrm{~B}$ & 0 & 11 & 27 & 4 \\
\hline \multicolumn{6}{|c|}{ Experiment III } \\
\hline 1 & CO39 & 0 & 27 & 20 & 0 \\
\hline 16 & IRBLks-F5 & 0 & 27 & 19 & 0 \\
\hline 17 & IRBLsh-S & 0 & 27 & 20 & 0 \\
\hline 18 & IRBLta-CP1 & 0 & 24 & 20 & 0 \\
\hline 19 & IRBL12-M & 0 & 23 & 17 & 0 \\
\hline 20 & Lement & 0 & 19 & 20 & 0 \\
\hline 21 & Dular & 0 & 24 & 20 & 0 \\
\hline 22 & $\mathrm{P} 183$ & 0 & 25 & 20 & 0 \\
\hline 23 & Mixture $^{\mathrm{a}}$ & 0 & 25 & 18 & 0 \\
\hline
\end{tabular}

${ }^{a}$ Containing an equal number of seeds of 14 japonica rice cultivars from America (Blue Belle and Peeso), Bulgaria (MC, Myxak, and 04-329), Italy (3224 and 8068), Japan (Akita Komochi, Toyonishiki, and 05-394), Korea (WL060097), and Russia (Спроги, Узрос 269, and UZ Ros 72). 
from 2539. However, we found that the avirulence of 2539 on a number of rice accessions (22 individual cultivars, including $\mathrm{CO} 39$, and a mixture of 14 cultivars) tested appeared to be controlled only by the AVRI-CO39 locus, according to the segregation of pathogenicity on these rice accessions in a genetic population derived from Guy11 × 2539. Similar results were also observed by Smith and Leong (1994). By inoculating progeny strains of Guy $11 \times 2539$ onto five near-isogenic lines (NIL) of $\mathrm{CO} 39$, each containing a different $R$ gene, they revealed that no other $A V R$ genes corresponding to the $R$ genes in these NIL were identified in 2539. We further proved, by an inoculation experiment with two transformed Guy11 strains, that all except one of the rice cultivars tested were resistant to the AVRICO39 gene. In regard to the only exception found in rice cv. Shen $3 \mathrm{~B}$, a possible explanation could be that there was another $A V R$ gene specific to Shen3B in 2539, which was tightly linked to (or located at the same locus as) AVR1-CO39 so that it segregated together with AVRI-CO39 completely in the genetic population investigated. Hence, based on the results of this study, it would be appropriate to draw a conclusion that the AVRI-CO39 locus is a predominant, if not the unique, locus and gene AVRl-CO39 is a predominant gene at the locus conferring the broad avirulence of 2539 on rice.

Previous studies have indicated that AVRI-CO39 is a specific $A V R$ gene of $M$. oryzae, which does not exist in other species of Magnaporthe (Tosa et al. 2005). AVRl-CO39 might be formed during the early evolution stage of the $M$. grisea complex (Tosa et al. 2005). However, its function has been lost due to mutations in all isolates from rice but retained in all isolates from other plant hosts (Farman et al. 2002; Tosa et al. 2005). Similar results were also obtained by Couch and associates (2005). This implies that the gene might be related to the divergence of host specificity with regard to rice between the subpopulation of the $M$. grisea complex from rice and that from other plant hosts. Combining the results of Farman and associates (2002), Tosa and associates (2005), and Couch and associates (2005), and those of Smith and Leong (1994) and this study, it would be reasonable to infer that the AVRI-CO39 locus is a species-wise instead of a cultivar-wise host-specific $A V R$ locus of $M$. oryzae for rice, and the specificity is predominantly controlled by gene AVRl-CO39 at the locus.

In the $1.05-\mathrm{kb}$ region covering the AVRl-CO39 gene, there are eight putative open reading frames (ORF), among which ORF3 encodes the largest predicted polypeptide (containing 89 amino acids) and is thought most likely to be the AVRICO39 gene (Leong 2008). To validate this prediction, we constructed an expression vector of ORF3 from 2539 (driven by the constitutive promoter RP27) and transferred it into Guy11. Two positive transformants, R3-1 and R3-2, were obtained. Inoculation experiment showed that these two transformants were all virulent to CO39 like the wild-type Guy11. Reversetranscription PCR analysis confirmed that ORF3 was expressed in the two transformants after inoculation. These results suggest that ORF3 is not sufficient to exhibit the avirulent function of AVR1-CO39. Hence, the functional sequence of AVR1-CO39 remains a puzzle.

An $R$ gene of rice interacting with AVR1-CO39, named as $\mathrm{Pi}$-CO39(t), has been fine mapped in cv. CO39 (Chauhan et al. 2002). However, exact molecular confirmation of the gene has not been reported. If the aforementioned inference about the host specificity of $A V R I-C O 39$ is correct, it can be expected that most rice cultivars must carry $\mathrm{Pi}-\mathrm{CO} 39(\mathrm{t})$. However, Tosa and associates (2005) reported that, among 33 rice accessions that were susceptible to PO12-7301-2 (an isolate of $M$. oryzae from rice lacking $A V R 1-C O 39$ ), 32 accessions (except for CO39) were unexpectedly also susceptible to PAS1-3-1 (a transformant of PO12-7301-2 carrying AVR1-CO39). Therefore, they suggested that $\mathrm{Pi}-\mathrm{CO} 39(\mathrm{t})$ should not be widely distributed in cultivated rice. This is obviously inconsistent with the result of our study, in which 35 of 36 rice cultivars showed resistance to transformed Guy11 strains carrying the AVRl-CO39 gene. In addition, this also seems to be contradictory to another result obtained by them in the same study (Tosa et al. 2005). They found that all of the 33 rice accessions tested were resistant to both GFSI1-7-2, the donor isolate from foxtail millet (Setaria italica) providing the functional AVR1-CO39 allele for PAS1$3-1$, and another isolate $(\mathrm{Br} 48)$ from wheat (Triticum aestivum) carrying a functional AVRl-CO39 homolog. This result appears to support the expectation that most rice cultivars carry $\mathrm{Pi}$ CO39(t). Certainly, this contradiction could be explained constrainedly by assuming that the two isolates GFSI1-7-2 and $\mathrm{Br} 48$ happened to carry one (or more) other AVR genes apart from $A V R 1-C O 39$, and the 32 rice accessions susceptible to PAS1-3-1 but resistant to GFSI1-7-2 and $\mathrm{Br} 48$ happened to carry the corresponding $R$ genes simultaneously. However, such an assumed situation appears to be very unlikely to occur as long as the 32 rice accessions tested were from unrelated sources. Further studies will be needed to make the issue clear.

\section{MATERIALS AND METHODS}

\section{M. oryzae strains.}

Strains 2539 and Guy11 were hybridized to construct a genetic population. This cross was previously used for the cloning of gene AVR1-CO39 (Farman and Leong 1998). Individual ascospores produced by the hybrid of $2539 \times$ Guy 11 were randomly isolated following the method of Valent and associates (1991). In the isolation of ascospores, hundreds of asci were mixed in sterilized water at $25^{\circ} \mathrm{C}$ for 2 to $3 \mathrm{~h}$ to allow ascospores to be released; then, individual ascospores were picked randomly. Because so many asci were mixed together, it would be very unlikely to isolate two ascospores from the same ascus.

\section{Detection of gene AVR1-CO39 in M. oryzae strains.}

DNA extraction from $M$. oryzae strains was performed following the method of Talbot and associates (1993). Detection of AVR1-CO39 in each strain was conducted following the PCR system of Tosa and associates (2005) with a pair of specific primers: AVF1-2 (5'-TGCCGCATTTTGCTAACC-3') and AVR1-2 (5'-GCGAATCCATAGACAAGGAC-3'). PCR products were electrophoresed in $1 \%$ agarose gel and stained with ethidium bromide.

\section{Transformation of Guy11 with gene $A V R 1-C O 39$ from 2539.}

Following the method of Tosa and associates (2005), a 1.70$\mathrm{kb}$ fragment containing the avirulence gene AVR1-CO39 was isolated from 2539 by PCR with a pair of specific primers: WF6 (5'-TAAACACCGCCTGAGATAGT-3') and WR4 (5'TGGTCACGGTTGGCAGTC- $3^{\prime}$ ), then inserted into plasmid pMD18-T (Takara). The recombinant plasmid carrying authentic AVR1-CO39 confirmed by sequencing and a plasmid pCX62 carrying a resistant gene to hygromycin B was used to cotransform Guy11. Transformants that could grow on the selective agar containing hygromycin B were transferred to potato dextrose agar media for secondary screening.

\section{Inoculation.}

Rice seed were pregerminated and then sown onto plastic pots filled with paddy field soil. Five rice cultivars (accessions) with 10 seeds each were planted in a pot. Inoculation of $M$. oryzae strains on rice seedlings was performed following the approach described by Smith and Leong (1994). Two replicates were set for each inoculation combination (an M. oryzae 
strain versus a rice cultivar). The two parental strains (Guy11 and 2539) were used as control. Lesions were evaluated following the system described by Valent and associates (1991). Inoculation on detached leaves was performed according to the system described by Liu and associates (2006).

\section{ACKNOWLEDGMENTS}

This work was supported by the National Natural Science Foundation of China (grant number 30671123) and the Commonweal Specialized Research Fund of China Agriculture (grant number 200803008). The rice seeds of accession numbers 16 to 23 were supplied by the Chinese Academy of Agricultural Sciences, Guangdong Academy of Agricultural Sciences, and Fujian Academy of Agricultural Sciences.

\section{LITERATURE CITED}

Bohnert, H. U., Fudal, I., Dioh, W., Tharreau, D., Notteghem, J. L., and Lebrun, M. H. 2004. A putative polyketide synthase/peptide synthetase from Magnaporthe grisea signals pathogen attack to resistant rice. Plant Cell 16:2499-2513.

Chauhan, R. S., Farman, M. L., Zhang, H. B., and Leong, S. A. 2002. Genetic and physical mapping of a rice blast resistance locus, Pi-CO39(t), that corresponds to the avirulence gene AVR1-CO39 of Magnaporthe grisea. Mol. Genet. Genomcis 267:603-612.

Couch, B. C., and Kohn, L. M. 2002. A multilocus gene genealogy concordant with host preference indicates segregation of a new species, Magnaporthe oryzae, from M. grisea. Mycologia 94:683-693.

Couch, B. C., Fudal, I., Lebrun, M. H., Tharreau, D., Valent, B., van Kim, P., Nottéghem, J.-L., and Kohn, L. M. 2005. Origins of host-specific populations of the blast pathogen Magnaporthe oryzae in crop domestication with subsequent expansion of pandemic clones on rice and weeds of rice. Genetics 170:613-630.

Farman, M. L., and Leong, S. A. 1998. Chromosome walking to the AVR1CO39 avirulence gene of Magnaporthe grisea: Discrepancy between the physical and genetic maps. Genetics 150:1049-1058.

Farman, M. L., Eto, Y., Nakao, T., Tosa, Y., Nakayashiki, H., Mayama, S., and Leong, S. A. 2002. Analysis of the structure of the AVR1-CO39 avirulence locus in virulent rice-infecting isolates of Magnaporthe grisea. Mol. Plant-Microbe Interact. 15:6-16.

Jia, Y., McAdams, S. A., Bryan, G. T., Hershey, H. P., and Valent, B. 2000. Direct interaction of resistance gene and avirulence gene products confers rice blast resistance. EMBO (Eur. Mol. Biol. Organ.) J. 19:40044014.

Leong, S. A. 2008. The ins and outs of host recognition of Magnaporthe oryzae. Pages 119-216 in: The Genomics of Disease. J. P. Gufstason, J. Taylor, and G. Stacey, eds. Springer Science Business Media, New York.

Leong, S. A., Farman, M., Smith, J., Budde, A., Tosa, Y., and Nitta, N.
1994. Molecular genetic approach to the study of cultivar specificity in the rice blast fungus, Pages 86-110 in: Rice Blast Disease. R. S. Zeigler, S. A. Leong, and P. S. Teng. CAB international/International Rice Research Institute, Oxon, U.K.

Leung, H., Borromeo, E. S., Bernado, M. A., and Notteghem, J. L. 1988. Genetic analysis of virulence in the rice blast fungus Magnaporthe grisea. Phytopathology 78:1227-1233.

Li, W., Wang, B., Wu, J., Lu, G., Hu, Y., Zhang, X., Zhang, Z., Zhao, Q., Feng, Q., Zhang, H., Wang, Z., Wang, G., Han, B., Wang, Z., and Zhou, B. 2009. The Magnaporthe oryzae avirulence gene AvrPiz-t encodes a predicted secreted protein that triggers the immunity in rice mediated by the blast resistance gene Piz-t. Mol. Plant-Microbe Interact. 22:411-420.

Liu, P., Wang, Z., Wang, Q., and Li, D. 2006. Agrobacterium tumefaciensmediated transformation of Magnaporthe grisea and identification of pathogenicity defective mutant. Chin. J. Rice Sci. 20:231-237.

Miki, S., Matsui, K., Kito, H., Otsuka, K., Ashizawa, T., Yasuda, N., Fukiya, S., Sato, J., Hirayae, K., Fujita, Y., Nakajima, T., Tomita, F., and Sone, T. 2009. Molecular cloning and characterization of the AVR-Pia locus from a Japanese field isolate of Magnaporthe oryzae. Mol. Plant Pathol. 10:361-374.

Orbach, M. J., Farrall, L., Sweigard, J. A., Chumley, F. G., and Valent, B. 2000. A telomeric avirulence gene determines efficacy for the rice blast resistance gene $\mathrm{Pi}$-ta. Plant Cell 12:2019-2032.

Silué, D., Nottéghem, J. L., and Tharreau, D. 1992. Evidence for a genefor-gene relationship in the Oryza sativa-Magnaporthe grisea pathosystem. Phytopathology 82:577-580.

Smith, J. R., and Leong, S. A. 1994. Mapping of a Magnaporthe grisea locus affecting rice (Oryza sativa) cultivar specificity. Theor. Appl. Genet. 88:901-908.

Sweigard, J. A., Carroll, A. M., Kang, S., Farrall, L., Chumley, F. G., and Valent, B. 1995. Identification, cloning, and characterization of PWL2, a gene for host species specificity in the rice blast fungus. Plant Cell 7:1221-1233.

Talbot, N. J., Salch, Y. P., Margery, M. A., and Hamer, J. E. 1993. Karyotypic variation within clonal lineages of the rice blast fungus, Magnaporthe grisea. Appl. Environ. Microbiol. 59:585-593.

Tosa, Y., Osue, J., Eto, Y., Oh, H. S., Nakayashiki, H., Mayama, S., and Leong, S. A. 2005. Evolution of an avirulence gene, AVR1-CO39, concomitant with the evolution and differentiation of Magnaporthe oryzae. Mol. Plant-Microbe Interact. 18:1148-1160.

Valent, B., Farrall, L., and Chumley, F. G. 1986. Genetic studies of fertility and pathogenicity in Magnaporthe grisea (Pyricularia oryzae). Iowa State J. Res. 60:569-594.

Valent, B., Leonard, F., and Forrest, G. C. 1991. Magnaporthe grisea genes for pathogenicity and virulence identified through a series of backcrosses. Genetics 127:87-101.

Yoshida, K., Saitoh, H., Fujisawa, S., Kanzaki, H., Matsumura, H., Yoshida, K., Tosa, Y., Chuma, I., Takano, Y., Win, J., Kamoun, S., and Terauchia, R. 2009. Association genetics reveals three novel avirulence genes from the rice blast fungal pathogen Magnaporthe oryzae. Plant Cell 21:1573-1591. 